PROCEEDINGS OF THE

AMERICAN MATHEMATICAL SOCIETY

Volume 131, Number 12, Pages 3807-3812

S 0002-9939(03)06962-4

Article electronically published on March 25, 2003

\title{
POWER BOUNDED OPERATORS AND SUPERCYCLIC VECTORS
}

\author{
V. MÜLLER
}

(Communicated by Joseph A. Ball)

\begin{abstract}
By the well-known result of Brown, Chevreau and Pearcy, every Hilbert space contraction with spectrum containing the unit circle has a nontrivial closed invariant subspace. Equivalently, there is a nonzero vector which is not cyclic.

We show that each power bounded operator on a Hilbert space with spectral radius equal to one has a nonzero vector which is not supercyclic. Equivalently, the operator has a nontrivial closed invariant homogeneous subset. Moreover, the operator has a nontrivial closed invariant positive cone.
\end{abstract}

Let $T$ be a bounded linear operator acting on a complex Banach space $X$. A vector $x \in X$ is called cyclic (supercyclic, hypercyclic) for $T$ if the set $\{p(T) x$ : $p$ polynomial $\}\left(\left\{\lambda T^{n} x: \lambda \in \mathbb{C}, n=0,1, \ldots\right\},\left\{T^{n} x: n=0,1, \ldots\right\}\right.$, respectively $)$ is dense in $X$.

Clearly, $T$ has a nontrivial closed invariant subspace (subset, homogeneous subset) if and only if there is a nonzero vector in $X$ which is not cyclic (hypercyclic, supercyclic, respectively); a subset $M \subset X$ is called homogeneous if $\mathbb{C} M \subset M$.

By the well-known example of Read $\left[\mathbb{R}\right.$, there is an operator on $\ell_{1}$ without nontrivial closed invariant subsets. For operators on Hilbert spaces no negative results are known; the best positive result is that each Hilbert space contraction whose spectrum contains the unit circle has a nontrivial closed invariant subspace $\mathrm{BCP}$.

The main result of this paper is that each Hilbert space contraction (or more generally power bounded operator) whose spectrum contains at least one point from the unit circle has a nontrivial closed invariant homogeneous subset. With more work we also show that the same result holds for Banach space operators of class $C_{00}$. In both cases there is also a nontrivial closed invariant positive cone.

Note that each power bounded operator has nontrivial closed invariant subsets since the orbit $\left\{T^{n} x: n=0,1, \ldots\right\}$ is bounded, and hence nondense, for each vector $x$.

All spaces considered in this paper are complex. For an operator $T$ acting on a Banach space $X$ denote its spectral radius by $r(T)$ and the essential spectrum by $\sigma_{e}(T)=\{\lambda \in \mathbb{C}: T-\lambda$ is not Fredholm $\}$.

Received by the editors June 19, 2002 and, in revised form, July 10, 2002.

1991 Mathematics Subject Classification. Primary 47A16, 47A15.

Key words and phrases. Supercyclic vector, invariant subspace problem, power bounded operator.

This research was supported by grant No. 201/03/0041 of GA ČR. 
We start with the following lemma.

Lemma 1. Let $K \geq 1$. Then there exist positive numbers $c_{i}(i \in \mathbb{N})$ such that $\sum_{i=1}^{\infty} c_{i}^{2}=1$ and $\sum_{i=k+1}^{\infty} c_{i}^{2}>3 K c_{k}$ for all $k \geq 1$.

Proof. Note first that

$$
\lim _{k \rightarrow \infty} k^{2 / 3} \sum_{i=k+1}^{\infty} i^{-4 / 3}=\infty .
$$

Indeed, we have $\sum_{i=k+1}^{\infty} i^{-4 / 3} \geq \int_{k+1}^{\infty} x^{-4 / 3} \mathrm{~d} x=3(k+1)^{-1 / 3}$, and so

$$
\lim _{k \rightarrow \infty} k^{2 / 3} \sum_{i=k+1}^{\infty} i^{-4 / 3} \geq \lim _{k \rightarrow \infty} \frac{3 k^{2 / 3}}{(k+1)^{1 / 3}}=\infty .
$$

By (1), there exists $k_{0}$ such that

$$
k^{2 / 3} \sum_{i=k+1}^{\infty} i^{-4 / 3}>3 K\left(\sum_{i=1}^{\infty} i^{-4 / 3}\right)^{1 / 2}
$$

for all $k \geq k_{0}$. For $j \in \mathbb{N}$ set

$$
c_{j}=\left(j+k_{0}\right)^{-2 / 3}\left(\sum_{i=k_{0}+1}^{\infty} i^{-4 / 3}\right)^{-1 / 2} .
$$

Then $\sum_{i=1}^{\infty} c_{i}^{2}=1$. Let $k \geq 1$. Then, by (2),

$$
\begin{aligned}
\sum_{i=k+1}^{\infty} c_{i}^{2} & =\frac{\sum_{i=k+1}^{\infty}\left(k_{0}+i\right)^{-4 / 3}}{\sum_{i=k_{0}+1}^{\infty} i^{-4 / 3}}>\frac{3 K\left(\sum_{i=1}^{\infty} i^{-4 / 3}\right)^{1 / 2}}{\sum_{i=k_{0}+1}^{\infty} i^{-4 / 3}} \cdot\left(k+k_{0}\right)^{-2 / 3} \\
& \geq \frac{3 K\left(k+k_{0}\right)^{-2 / 3}}{\left(\sum_{i=k_{0}+1}^{\infty} i^{-4 / 3}\right)^{1 / 2}}=3 K c_{k} .
\end{aligned}
$$

The next result, which is of independent interest, is a generalization of [M2], Corollary 3.4.

Theorem 2. Let $T$ be an operator acting on a Hilbert space $H$ such that $1 \in \sigma(T)$ and $T^{n} x \rightarrow 0$ for all $x \in H$. Let $\left(a_{n}\right)_{n=1}^{\infty}$ be a sequence of positive numbers such that $\lim _{n \rightarrow \infty} a_{n}=0$ and $\sup a_{n}<1$. Then there exists $x \in H$ of norm one such that $\operatorname{Re}\left\langle T^{n} x, x\right\rangle>a_{n}$ for all $n \geq 1$.

Proof. By the Banach-Steinhaus theorem, $\sup _{n}\left\|T^{n}\right\|<\infty$. Let $K=\sup _{n}\left\|T^{n}\right\|$. Clearly $K \geq 1$ and $r(T)=1$.

Suppose first that $1 \notin \sigma_{e}(T)$. Then 1 is an eigenvalue of $T$ and there exists $x \in H$ of norm one such that $T x=x$. Then $\operatorname{Re}\left\langle T^{n} x, x\right\rangle=1$ for all $n$.

Let $1 \in \sigma_{e}(T)$. Then $1 \in \partial \sigma_{e}(T)$, and so $T-I$ is not upper semi-Fredholm; see [HW]. Consequently, for all $\varepsilon>0$ and $M \subset H$ with $\operatorname{codim} M<\infty$ there exists $u \in M$ of norm one such that $\|T u-u\|<\varepsilon$. Moreover, given $n_{0} \in \mathbb{N}$, we can also find $v \in M$ of norm one such that $\left\|T^{j} v-v\right\|<\varepsilon$ for all $j \leq n_{0}$. 
Replacing the numbers $a_{n}$ by $\sup \left\{a_{i}: i \geq n\right\}$ we can assume without loss of generality that $1>a_{1} \geq a_{2} \geq \cdots$. By Lemma 1 , there are positive numbers $c_{i}$ such that $\sum_{i=1}^{\infty} c_{i}^{2}=1$ and $\sum_{i=k+1}^{\infty} c_{i}^{2}>3 K c_{k}$ for all $k \geq 1$.

For $i=1,2, \ldots$ let $\delta_{i}$ be a positive number satisfying $\delta_{i}<\frac{1-a_{1}}{2^{i}}$ and $\delta_{i}<$ $\min \left\{\frac{K c_{k}}{i \cdot 2^{i-k+1}}: k=1, \ldots, i+1\right\}$.

Find $m_{0} \in \mathbb{N}$ such that $a_{m_{0}}<\sum_{i=2}^{\infty} c_{i}^{2}-3 K c_{1}$. We construct inductively an increasing sequence $\left(m_{i}\right)_{i=0}^{\infty}$ of positive integers and a sequence $\left(x_{i}\right)_{i=1}^{\infty} \subset H$ in the following way:

Let $k \in \mathbb{N}$ and suppose that $x_{i} \in H$ and $m_{i}$ have already been constructed for all $i<k$. Choose $x_{k} \in X$ of norm one such that

$$
x_{k} \perp T^{j} x_{i} \quad\left(i<k, 0 \leq j \leq m_{k-1}\right)
$$

and

$$
\left\|T^{j} x_{k}-x_{k}\right\|<\delta_{k} \quad\left(j \leq m_{k-1}\right) .
$$

Find $m_{k}>m_{k-1}$ such that

$$
\left\|T^{j} x_{i}\right\|<\delta_{k} \quad\left(i \leq k, j \geq m_{k}\right)
$$

and

$$
a_{m_{k}}<\sum_{i=k+2}^{\infty} c_{i}^{2}-3 K c_{k+1} .
$$

Suppose that $x_{i}$ and $m_{i}$ have been constructed in the above described way. Set $x=\sum_{i=1}^{\infty} c_{i} x_{i}$. Since $\left(x_{i}\right)$ is an orthonormal sequence, we have $\|x\|=\left(\sum_{i=1}^{\infty} c_{i}^{2}\right)^{1 / 2}$ $=1$.

For $n \leq m_{0}$ we have

$$
\begin{aligned}
& \operatorname{Re}\left\langle T^{n} x, x\right\rangle=\operatorname{Re} \sum_{i=1}^{\infty} c_{i}\left\langle T^{n} x_{i}, x\right\rangle=\operatorname{Re} \sum_{i=1}^{\infty} c_{i}\left(\left\langle x_{i}, x\right\rangle-\left\langle x_{i}-T^{n} x_{i}, x\right\rangle\right) \\
& \geq \sum_{i=1}^{\infty} c_{i}^{2}-\sum_{i=1}^{\infty} c_{i}\left\|x_{i}-T^{n} x_{i}\right\| \geq 1-\sum_{i=1}^{\infty} c_{i} \delta_{i}>1-\sum_{i=1}^{\infty} \frac{1-a_{1}}{2^{i}}=a_{1} \geq a_{n} .
\end{aligned}
$$

Let $k \geq 1$ and $m_{k-1}<n \leq m_{k}$. Then

$$
\begin{aligned}
& \operatorname{Re}\left\langle T^{n} x, x\right\rangle=\operatorname{Re} \sum_{i=1}^{k-1} c_{i}\left\langle T^{n} x_{i}, x\right\rangle+\operatorname{Re} c_{k}\left\langle T^{n} x_{k}, x\right\rangle+\operatorname{Re} \sum_{i=k+1}^{\infty} c_{i}\left\langle T^{n} x_{i}, x\right\rangle \\
& \geq-\sum_{i=1}^{k-1} c_{i}\left\|T^{n} x_{i}\right\|-K c_{k}+\operatorname{Re} \sum_{i=k+1}^{\infty} c_{i}\left(\left\langle x_{i}, x\right\rangle-\left\langle x_{i}-T^{n} x_{i}, x\right\rangle\right) \\
& \geq-\sum_{i=1}^{k-1} c_{i} \delta_{k-1}-K c_{k}+\sum_{i=k+1}^{\infty} c_{i}^{2}-\sum_{i=k+1}^{\infty} c_{i}\left\|x_{i}-T^{n} x_{i}\right\| \\
& \geq-(k-1) \delta_{k-1}-K c_{k}+\sum_{i=k+1}^{\infty} c_{i}^{2}-\sum_{i=k+1}^{\infty} \delta_{i} \geq \sum_{i=k+1}^{\infty} c_{i}^{2}-3 K c_{k}>a_{m_{k-1}} \geq a_{n}
\end{aligned}
$$

Thus $\operatorname{Re}\left\langle T^{n} x, x\right\rangle>a_{n}$ for all $n \geq 1$.

Theorem 3. Let $T$ be a power bounded operator on a Hilbert space $H$ with $\operatorname{dim} H \geq$ 2. Suppose that $r(T)=1$. Then there exists a nonzero vector $x \in H$ which is not 
supercyclic for T. Moreover, there exists a nontrivial closed positive cone invariant with respect to $T$.

Proof. Without loss of generality we may assume that $T$ is not a scalar multiple of the identity and that $1 \in \sigma(T)$. We also may assume that the point spectrum of $T^{*}$ is empty since $\overline{(T-\bar{\alpha}) H}=\operatorname{ker}\left(T^{*}-\alpha\right)^{\perp}$ is a nontrivial closed invariant subspace for each eigenvalue $\alpha$ of $T^{*}$.

We use the standard reduction of the problem; see $\left[\mathrm{NF}\right.$. Set $X_{1}=\{x \in H$ : $\left.T^{n} x \rightarrow 0\right\}$ and $X_{2}=\left\{x \in H: T^{* n} x \rightarrow 0\right\}$. Then $X_{1}$ is a closed subspace invariant with respect to $T$ and $X_{2}$ is a closed subspace invariant with respect to $T^{*}$. So $X_{2}^{\perp}$ is invariant with respect to $T$. Thus we may assume that both $X_{1}$ and $X_{2}$ are trivial (equal either to $\{0\}$ or to $H$ ).

If $X_{1}=\{0\}=X_{2}$, then $T$ is of class $C_{11}$ and therefore has plenty of invariant subspaces; see [NF], Theorem I.5.4. Thus we may assume that either $X_{1}=H$ (in this case $T^{n} x \rightarrow 0$ for all $x \in H$ ) or $X_{2}=H$ (and so $T^{* n} x \rightarrow 0$ for all $x$ ). By Theorem 2, in both cases there exists $x \in H$ of norm one such that $\operatorname{Re}\left\langle T^{n} x, x\right\rangle \geq 0$ for all $n \geq 0$. Hence $\operatorname{Re}\left\langle t T^{n} x, x\right\rangle \geq 0$ for all $t>0$ and $n \in \mathbb{N}$, and so the set $\left\{t T^{n} x: t>0, n=0,1, \ldots\right\}$ is not dense in $H$. By [LM], this implies that $x$ is not supercyclic for $T$.

Clearly, the set $\left\{t T^{n} x: t>0, n=0,1, \ldots\right\}$ is a nontrivial closed positive cone invariant with respect to $T$.

The situation for operators on Banach spaces is more complicated since the geometry in general Banach space is not so regular. We are able to obtain the main result for operators of class $C_{00}$, i.e., for operators $T: X \rightarrow X$ satisfying $T^{n} x \rightarrow 0$ and $T^{* n} x^{*} \rightarrow 0$ for all $x \in X$ and $x^{*} \in X^{*}$.

Lemma 4. Let $K \geq 1$. Then there are positive numbers $c_{i} \quad(i \geq 1)$ satisfying $\sum_{i=1}^{\infty} c_{i}<1 / 3$ and $\sum_{i=k+1}^{\infty} c_{i}^{2}>3 K c_{k}^{2}$ for all $k \geq 1$.

Proof. For $j=1,2, \ldots$ set $c_{j}=(j+18 K)^{-2}$. For $k \geq 1$ we have

$$
\begin{aligned}
& \sum_{i=k+1}^{\infty} c_{i}^{2}=\sum_{i=k+1}^{\infty}(i+18 K)^{-4} \geq \int_{k+1}^{\infty}(x+18 K)^{-4} \mathrm{~d} x \\
& =\frac{1}{3(k+1+18 K)^{3}}>\frac{1}{6(k+18 K)^{3}}>\frac{18 K}{6(k+18 K)^{4}}=3 K c_{k}^{2} .
\end{aligned}
$$

Similarly,

$$
\sum_{i=1}^{\infty} c_{i} \leq \int_{0}^{\infty}(x+18 K)^{-2} \mathrm{~d} x=\frac{1}{18 K}<1 / 3
$$

Theorem 5. Let $T$ be an operator on a Banach space $X$ such that $1 \in \sigma(T)$, $T^{n} x \rightarrow 0 \quad(x \in X)$ and $T^{* n} x^{*} \rightarrow 0 \quad\left(x^{*} \in X^{*}\right)$. Let $\left(a_{i}\right)$ be a sequence of positive numbers such that $\lim _{i \rightarrow \infty} a_{i}=0$. Then there are $x \in X$ and $x^{*} \in X^{*}$ such that $\operatorname{Re}\left\langle T^{n} x, x^{*}\right\rangle>a_{n}$ for all $n \geq 1$.

Proof. Replacing $a_{n}$ by $\sup \left\{a_{i}: i \geq n\right\}$ we may assume that $a_{1} \geq a_{2} \geq \cdots$.

By the Banach-Steinhaus theorem, $T$ is power bounded. Let $K=\sup _{n}\left\|T^{n}\right\|$. Clearly $K \geq 1$ and $r(T)=1$.

If $1 \notin \sigma_{e}(T)$, then 1 is an eigenvalue of $T$. Let $x \in H$ be a corresponding eigenvector and let $x^{*} \in X^{*}$ satisfy $\left\langle x, x^{*}\right\rangle=1$. Then $\operatorname{Re}\left\langle T^{n} x, x^{*}\right\rangle=1$ for all $n$. 
In the following we suppose that $1 \in \sigma_{e}(T)$. As in Theorem 2, T-I is not upper semi-Fredholm. Consequently, for all $\varepsilon>0, n_{0} \in \mathbb{N}$ and $M \subset H$ with $\operatorname{codim} M<\infty$ there exists $u \in M$ of norm one such that $\left\|T^{j} u-u\right\|<\varepsilon$ for all $j \leq n_{0}$.

By Lemma 4 , there are positive numbers $c_{i}$ such that $\sum_{i=k+1}^{\infty} c_{i}^{2}>3 K c_{k}^{2}$ for all $k \geq 1$ and $\sum_{i=1}^{\infty} c_{i} \leq 1 / 3$.

For $i=1,2, \ldots$ set $\delta_{i}=\min \left\{\frac{c_{k}^{2}}{2^{i-k+5}}: k=1, \ldots, i+1\right\}$.

Choose $m_{0}$ such that $a_{m_{0}}<\sum_{i=2}^{\infty} c_{i}^{2}-3 K c_{1}^{2}$.

We construct inductively an increasing sequence $\left(m_{i}\right)$ of positive integers and sequences $\left(x_{i}\right) \subset X,\left(x_{i}^{*}\right) \subset X^{*}$.

Let $k \geq 1$ and suppose that $m_{i}, x_{i}$ and $x_{i}^{*}$ have already been constructed for $i \leq k-1$. Let $F_{k}=\bigvee\left\{T^{j} x_{i}: 1 \leq i \leq k-1,0 \leq j \leq m_{k-1}\right\}$. Clearly, $\operatorname{dim} F_{k}<\infty$ and, by [M1], Lemma 1, there is a subspace $M_{k} \subset X$ of finite codimension such that $\|f+m\| \geq \frac{2}{5}\|m\|$ for all $f \in F_{k}, m \in M_{k}$.

Choose $x_{k} \in M_{k} \cap \bigcap_{i=1}^{k-1} \operatorname{ker} x_{i}^{*}$ such that $\left\|x_{k}\right\|=1$ and $\left\|T^{j} x_{k}-x_{k}\right\| \leq \delta_{k} \quad(j \leq$ $\left.m_{k-1}\right)$.

Let $x_{k}^{*}$ be the functional on $F_{k} \vee\left\{x_{k}\right\}$ defined by $\left\langle f+\alpha x_{k}, x_{k}^{*}\right\rangle=\alpha$ for all $f \in F_{k}, \alpha \in \mathbb{C}$. We have $\left|\left\langle f+\alpha x_{k}, x_{k}^{*}\right\rangle\right|=|\alpha| \leq \frac{5}{2}\left\|f+\alpha x_{k}\right\|$. Hence $\left\|x_{k}^{*}\right\| \leq 5 / 2$ and, by the Hahn-Banach theorem, we can extend $x_{k}^{*}$ to a functional on $X$ (denoted by the same symbol $x_{k}^{*}$ ) with the same norm.

Find $m_{k}$ such that $\left\|T^{j} x_{i}\right\|<\delta_{k}$ and $\left\|T^{* j} x_{i}^{*}\right\|<\delta_{k}$ for all $j \geq m_{k}$ and $i=1, \ldots, k$, and $a_{m_{k}}<\sum_{i=k+1}^{\infty} c_{i}^{2}-3 K c_{k}^{2}$.

Let $\left(x_{i}\right),\left(x_{i}^{*}\right)$ and $\left(m_{i}\right)$ be the sequences constructed in the above-described way. Note that $\left\langle x_{i}, x_{j}^{*}\right\rangle=\delta_{i, j}$ (the Kronecker symbol) for all $i, j \geq 1$. Set $u=\sum_{i=1}^{\infty} c_{i} x_{i}$ and $u^{*}=\sum_{i=1}^{\infty} c_{i} x_{i}^{*}$. Then $\|u\| \leq \sum_{i=1}^{\infty} c_{i} \leq 1$ and $\left\|u^{*}\right\| \leq \sum_{i=1}^{\infty} \frac{5}{2} c_{i} \leq 1$.

Let $k \geq 1$ and $m_{k-1}<n \leq m_{k}$. Then

$$
\begin{gathered}
\operatorname{Re}\left\langle T^{n} u, u^{*}\right\rangle=\operatorname{Re} \sum_{i=1}^{k-1} c_{i}\left\langle T^{n} x_{i}, u^{*}\right\rangle+\operatorname{Re}\left\langle c_{k} x_{k}, \sum_{i=1}^{k-1} c_{i} T^{* n} x_{i}^{*}\right\rangle+\operatorname{Re}\left\langle c_{k} T^{n} x_{k}, c_{k} x_{k}^{*}\right\rangle \\
+\operatorname{Re}\left\langle c_{k} T^{n} x_{k}, \sum_{i=k+1}^{\infty} c_{i} x_{i}^{*}\right\rangle+\operatorname{Re} \sum_{i=k+1}^{\infty} c_{i}\left\langle T^{n} x_{i}, u^{*}\right\rangle \geq-\sum_{i=1}^{k-1} c_{i}\left\|T^{n} x_{i}\right\| \\
-c_{k} \sum_{i=1}^{k-1} c_{i}\left\|T^{* n} x_{i}^{*}\right\|-\frac{5}{2} K c_{k}^{2}+\operatorname{Re} \sum_{i=k+1}^{\infty} c_{i}\left(\left\langle x_{i}, u^{*}\right\rangle-\left\langle x_{i}-T^{n} x_{i}, u^{*}\right\rangle\right) \\
\geq-2 \delta_{k-1}-\frac{5}{2} K c_{k}^{2}+\sum_{i=k+1}^{\infty} c_{i}^{2}-\sum_{i=k+1}^{\infty} c_{i} \delta_{i} \geq \sum_{i=k+1}^{\infty} c_{i}^{2}-3 K c_{k}^{2}>a_{m_{k-1}} \geq a_{n} .
\end{gathered}
$$

For $n \leq m_{0}$ we have

$$
\begin{aligned}
& \operatorname{Re}\left\langle T^{n} u, u^{*}\right\rangle=\operatorname{Re} \sum_{i=1}^{\infty} c_{i}\left\langle T^{n} x_{i}, u^{*}\right\rangle \geq \operatorname{Re} \sum_{i=1}^{\infty} c_{i}\left\langle x_{i}, u^{*}\right\rangle-\operatorname{Re} \sum_{i=1}^{\infty} c_{i}\left\langle x_{i}-T^{n} x_{i}, u^{*}\right\rangle \\
& \geq \sum_{i=1}^{\infty} c_{i}^{2}-\sum_{i=1}^{\infty} c_{i} \delta_{i} \geq \sum_{i=1}^{\infty} c_{i}^{2}-\sum_{i=1}^{\infty} \frac{c_{i}^{2}}{2}>0 .
\end{aligned}
$$

Thus for certain positive multiples $x$ of $u$ and $x^{*}$ of $u^{*}$ we have $\operatorname{Re}\left\langle T^{n} x, x^{*}\right\rangle>a_{n}$ for all $n \geq 1$. 
Corollary 6. Let $T$ be an operator of class $C_{00}$ acting on a Banach space $X$ such

that $r(T)=1$. Then there exists a nonzero vector $x \in X$ which is not supercyclic for $T$.

\section{REFERENCES}

[BCP] S. Brown, B. Chevreau, C. Pearcy, On the structure of contraction operators II, J. Funct. Anal. 76 (1988), 30-55. MR 90b:47030b

[HW] R. Harte, A. Wickstead, Upper and lower Fredholm spectra II, Math. Z. 154 (1977), 253256. MR 56:12926]

[LM] F. Leon, V. Müller, Rotations of hypercyclic operators (to appear).

[M1] V. Müller, Local behaviour of the polynomial calculus of operators, J. Reine Angew. Math. 430 (1992), 61-68. MR 94b:47004]

[M2] - Orbits, weak orbits and local capacity of operators, Integral Equations Operator Theory 41 (2000), 230-253. MR 2002g:47009

[NF] B. Sz.-Nagy, C. Foias, Harmonic Analysis of Operators, Akadémiai Kiadó/North Holland, Budapest/Amsterdam, 1970. MR 43:947

[R] C.J. Read, The invariant subspace problem for a class of Banach spaces II. Hypercyclic operators, Israel J. Math. 63 (1988), 1-40. MR 90b:47013

Mathematical Institute, Czech Academy of Sciences, Zitna 25, 11567 Prague 1, Czech REPUBLIC

E-mail address: muller@math.cas.cz 